\title{
Steps Along the Path to Dihydrogen Activation at [FeFe] Hydrogenase Structural Models: Dependence of the Core Geometry on Electrocatalytic Proton Reduction
}

Mun Hon Cheah, Stacey J. Borg and Stephen P. Best*

School of Chemistry University of Melbourne, Victoria, Australia, 3010.

\section{Supplementary information:}

Author list for reference 26.

Fig. S1 SEC of reduction and reoxidation of $3 P$ in $\mathrm{THF} / \mathrm{TBAPF}_{6}$ under $0.275 \mathrm{MPa} \mathrm{Ar}$

Fig. S2 SEC of reduction and reoxidation of $3 \mathrm{P}$ in $\mathrm{CH}_{3} \mathrm{CN} / \mathrm{TBAPF}_{6}$ under $0.275 \mathrm{MPa} \mathrm{Ar}$

Fig. S3 Room temperature EPR spectrum of $\mathbf{3} \mathbf{P}^{\mathbf{1 -}}$.

Fig. S4 Fits of the EXAFS of $3 \mathbf{P}$ and $3 \mathbf{P}^{2-}$.

Fig. S5 Comparison between the observed and DFT-calculated IR spectra of $\mathbf{3 P}, \mathbf{3} \mathbf{P}^{\mathbf{1 -}}$ and $3 \mathbf{P}^{2-}$.

Fig. S6 Comparison of the IR spectra of species $\mathrm{H}_{2} 3 \mathbf{P}$ and DFT calculated spectra for the different isomers of $\left[\mathrm{H}_{2} 3 \mathbf{P}_{\mathbf{M}}\right]$.

Table S1 Details of the models and refinement parameters for the EXAFS fitting of $\mathbf{3 P}$ and $3 \mathbf{P}^{2-}$.

Table S2 Final values of the Fe-Fe distance obtained for EXAFS fitting of mixtures of $\mathbf{3 P}, \mathbf{3 P}^{\mathbf{1}^{-}}$ and $3 \mathbf{P}^{2-}$.

Table S3 Details of the simulation parameters used in calculation of the cyclic voltammetry of 3P with 0 to 6 equivalents of HOTs.

Table S4 Summary of the observed and calculated $v(\mathrm{CO})$ band intensities and wavenumbers. 
Reference 26: Frisch, M. J.; Trucks, G. W.; Schlegel, H. B.; Scuseria, G. E.; Robb, M. A.; Cheeseman, J. R.; Montgomery, J., J. A.; Vreven, T.; Kudin, K. N.; Burant, J. C.; Millam, J. M.; Iyengar, S. S.; Tomasi, J.; Barone, V.; Mennucci, B.; Cossi, M.; Scalmani, G.; Rega, N.; Petersson, G. A.; Nakatsuji, H.; Hada, M.; Ehara, M.; Toyota, K.; Fukuda, R.; Hasegawa, J.; Ishida, M.; Nakajima, T.; Honda, Y.; Kitao, O.; Nakai, H.; Klene, M.; Li, X.; Knox, J. E.; Hratchian, H. P.; Cross, J. B.; Bakken, V.; Adamo, C.; Jaramillo, J.; Gomperts, R.; Stratmann, R. E.; Yazyev, O.; Austin, A. J.; Cammi, R.; Pomelli, C.; Ochterski, J. W.; Ayala, P. Y.; Morokuma, K.; Voth, G. A.; Salvador, P.; Dannenberg, J. J.; Zakrzewski, V. G.; Dapprich, S.; Daniels, A. D.; Strain, M. C.; Farkas, O.; Malick, D. K.; Rabuck, A. D.; Raghavachari, K.; Foresman, J. B.; Ortiz, J. V.; Cui, Q.; Baboul, A. G.; Clifford, S.; Cioslowski, J.; Stefanov, B. B.; Liu, G.; Liashenko, A.; Piskorz, P.; Komaromi, I.; Martin, R. L.; Fox, D. J.; Keith, T.; AlLaham, M. A.; Peng, C. Y.; Nanayakkara, A.; Challacombe, M.; Gill, P. M. W.; Johnson, B.; Chen, W.; Wong, M. W.; Gonzalez, C.; Pople, J. A. Gaussian 03, Revision B.04, Gaussian, Inc.: Wallingford CT, 2004.

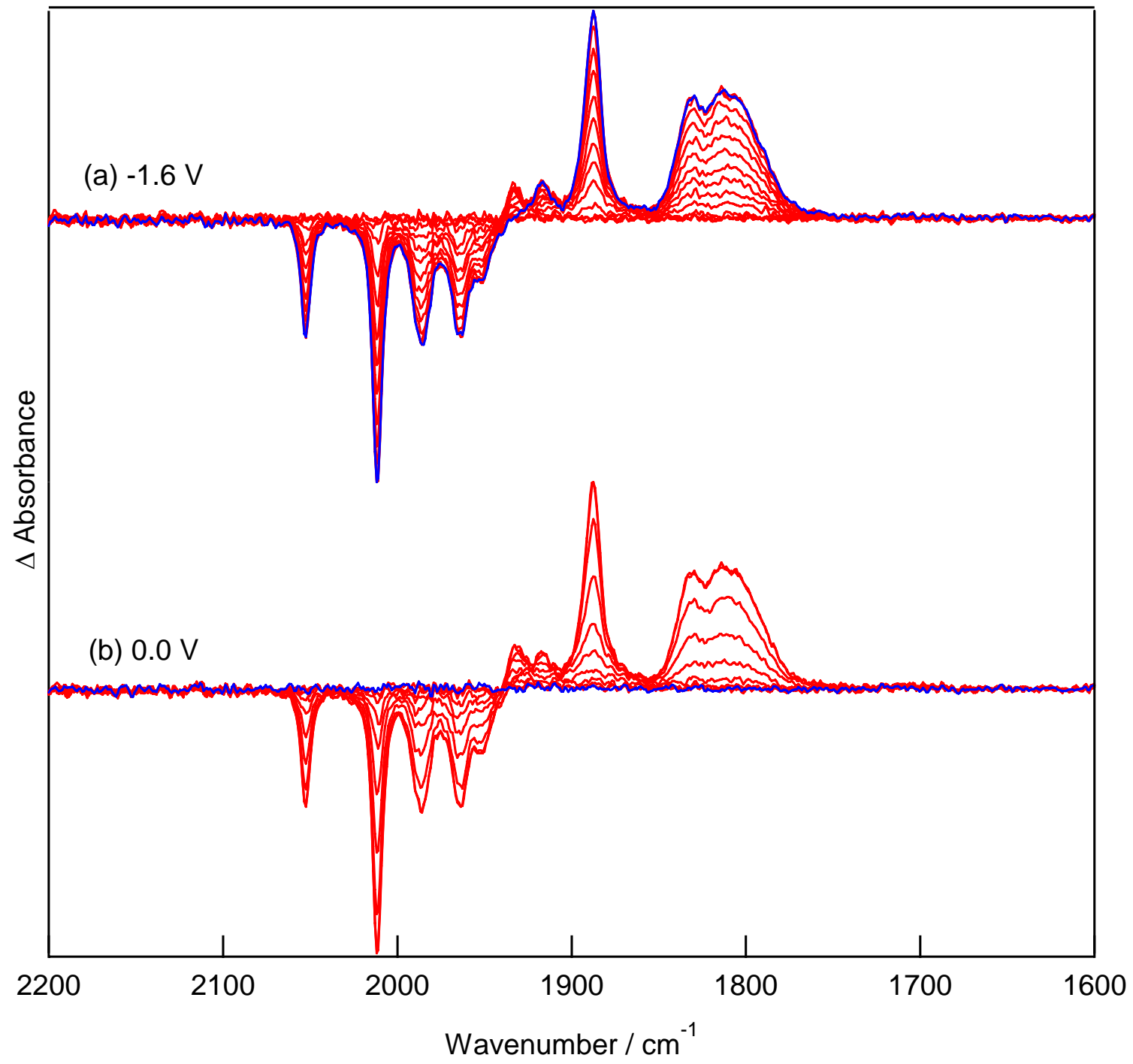

Figure S1. (a) Differential absorption spectra of a $3.3 \mathrm{mM}$ solution of $3 \mathbf{P}$ in $0.2 \mathrm{M} \mathrm{TBAPF}_{6} / \mathrm{THF}$ from 0 to $-1.6 \mathrm{~V}$. (b) Differential absorption spectra of same film of solution in (a) from -1.6 to $0 \mathrm{~V}$. 


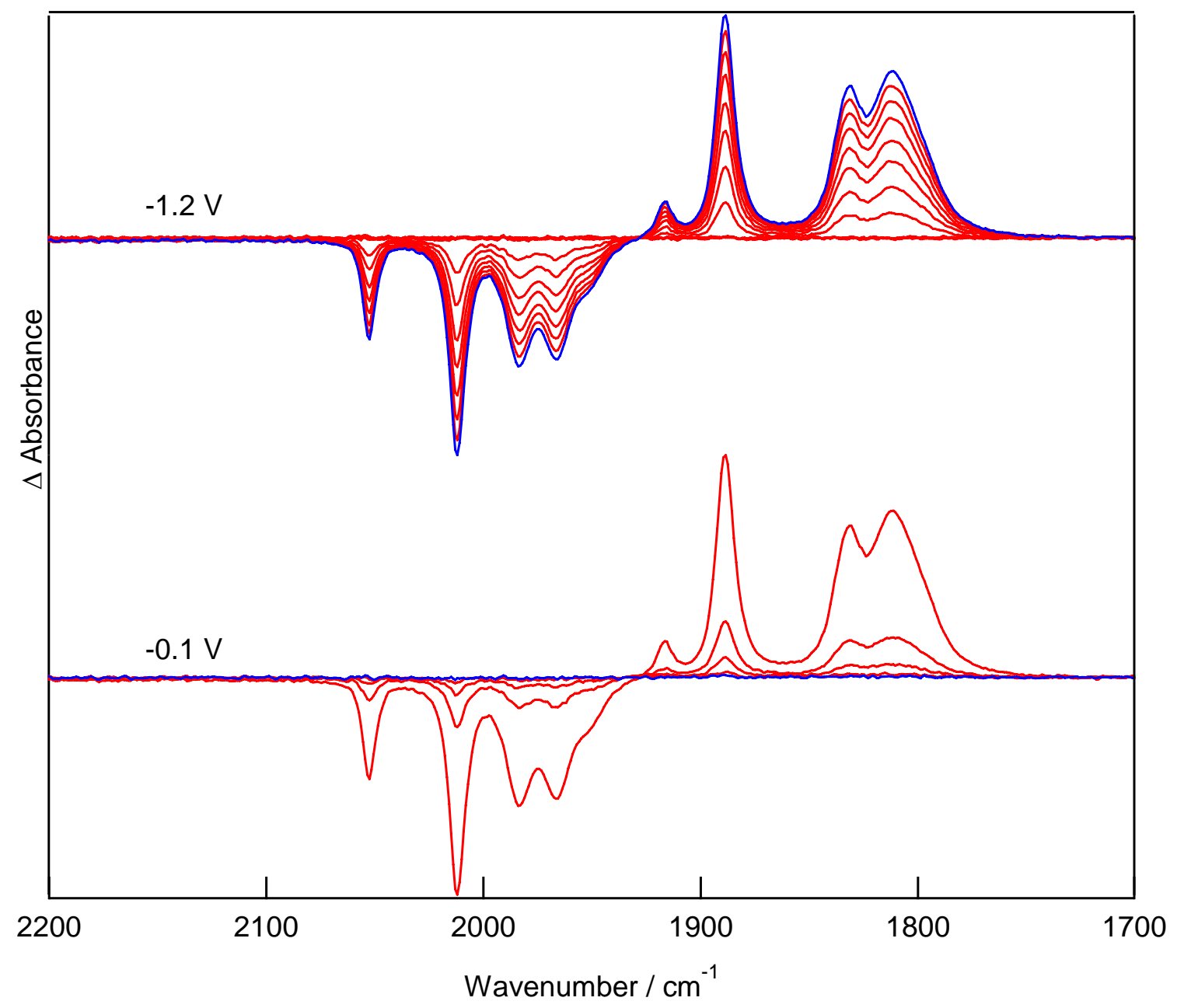

Figure S2. (a) Differential absorption spectra of a $4.5 \mathrm{mM}$ solution of $3 \mathbf{P}$ in $0.2 \mathrm{M} \mathrm{TBAPF} / \mathrm{CH}_{3} \mathrm{CN}$ from 0 to $-1.21 \mathrm{~V}$. (b) Differential absorption spectra of same film of solution in (a) from -1.21 to $0.1 \mathrm{~V}$. Note the absence of the bands due to $3 \mathbf{P}^{1-}$ and the presence of a reasonably well-defined isosbestic point. 


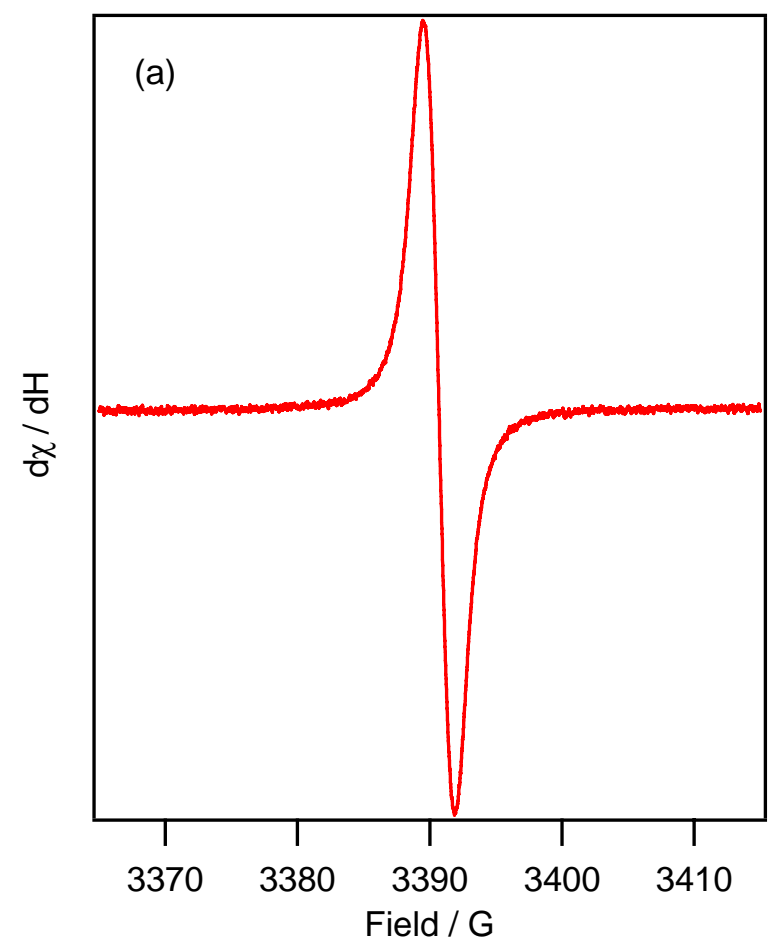

Figure S3 Room temperature EPR spectrum of $3 \mathbf{P}^{1-}$ generated by continuous-flow electrosynthesis. 

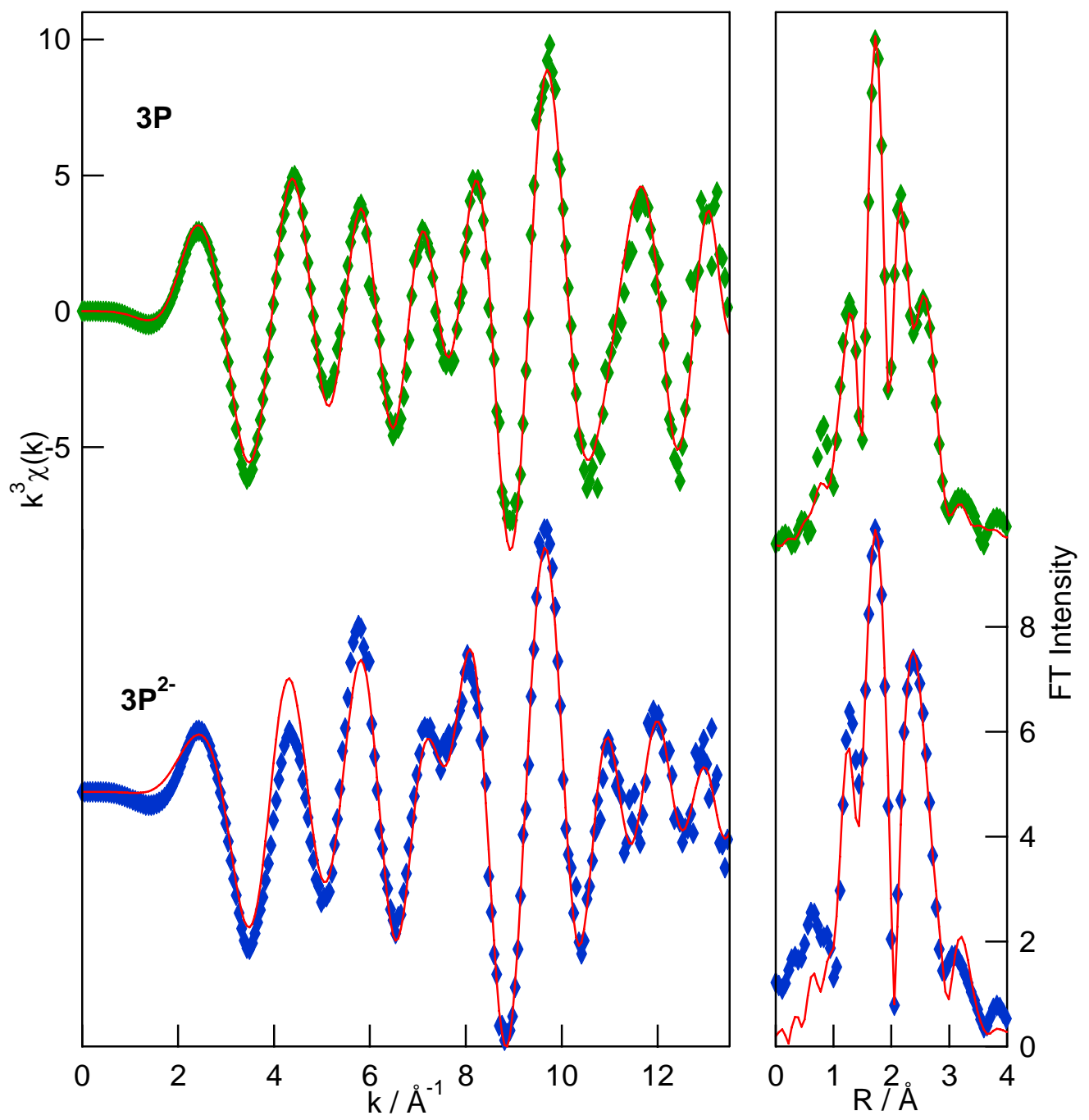

Figure S4. Fits of the EXAFS of $\mathbf{3 P}$ and $\mathbf{3} \mathbf{P}^{2-}$ and IR spectra of samples used for EXAFS (below)

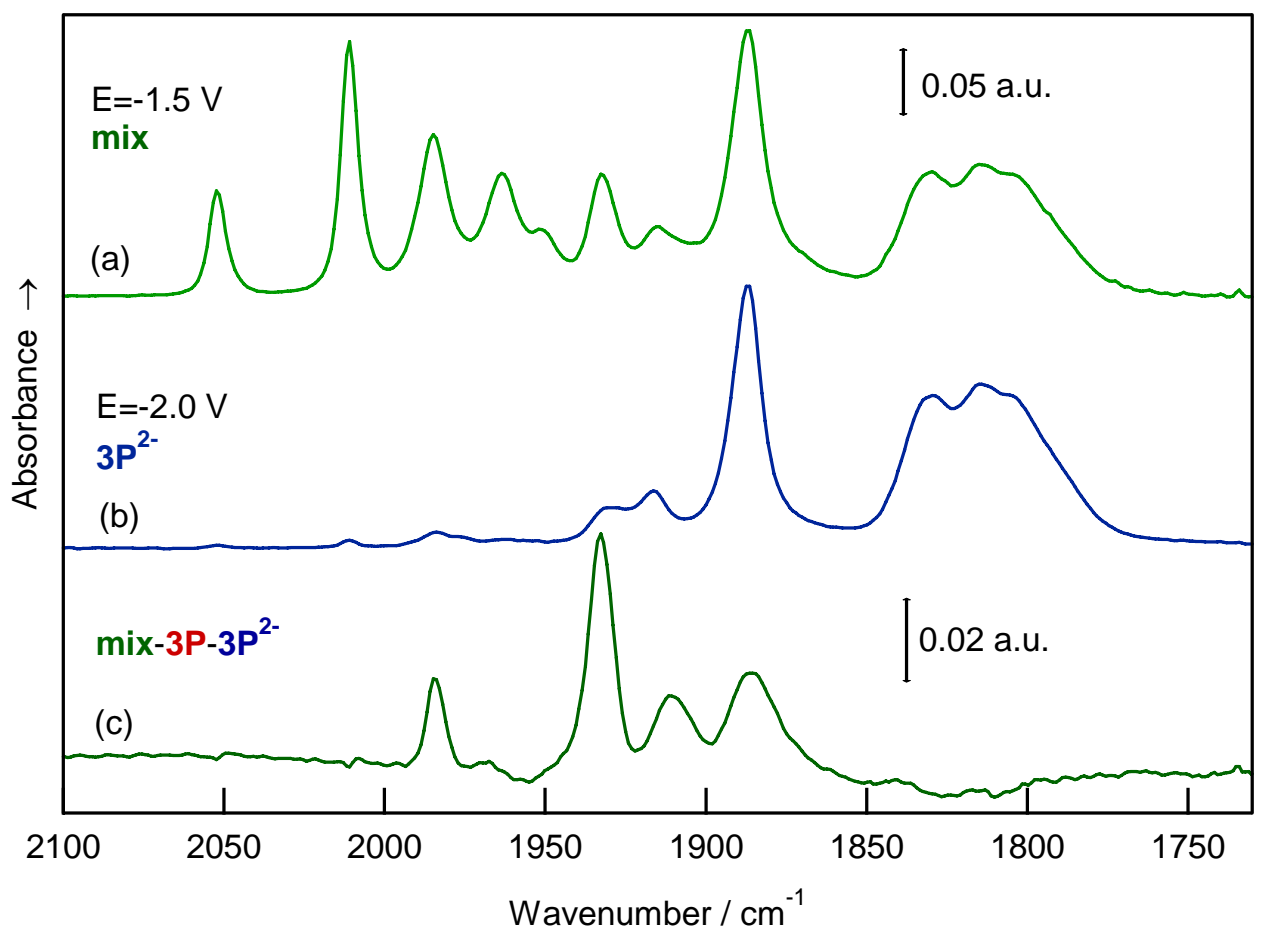




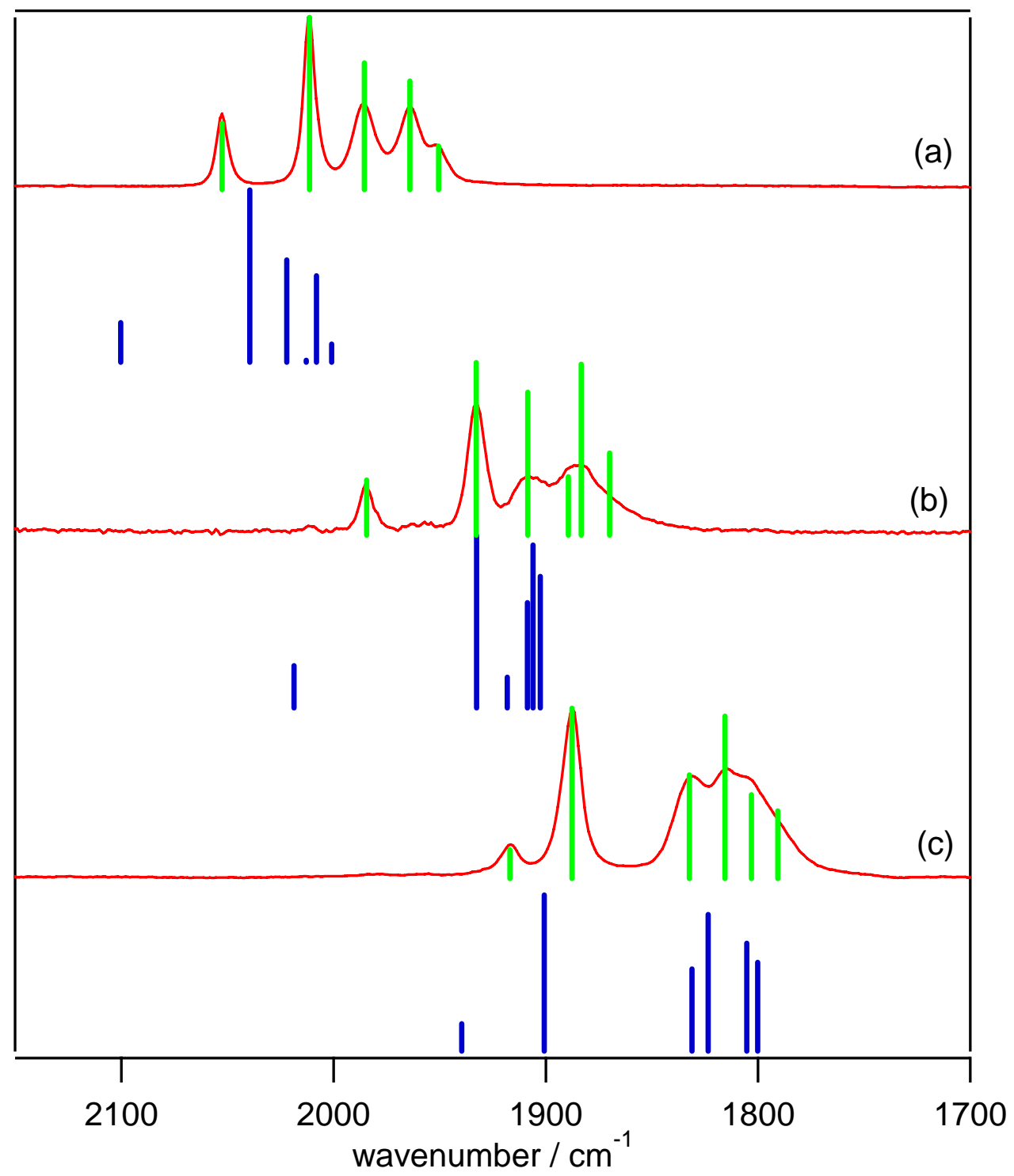

Figure S5. Comparison of calculated and experimental $v(\mathrm{CO})$ frequencies and relative intensities of (a) $3 \mathbf{P}$, , (b) $3 \mathbf{P}^{\mathbf{1}^{-}}$, (c) $3 \mathbf{P}^{2-}$. Top green lines are frequencies and intensities obtained by curve-fitting experimental spectra. Bottom blue lines are DFT calculations 


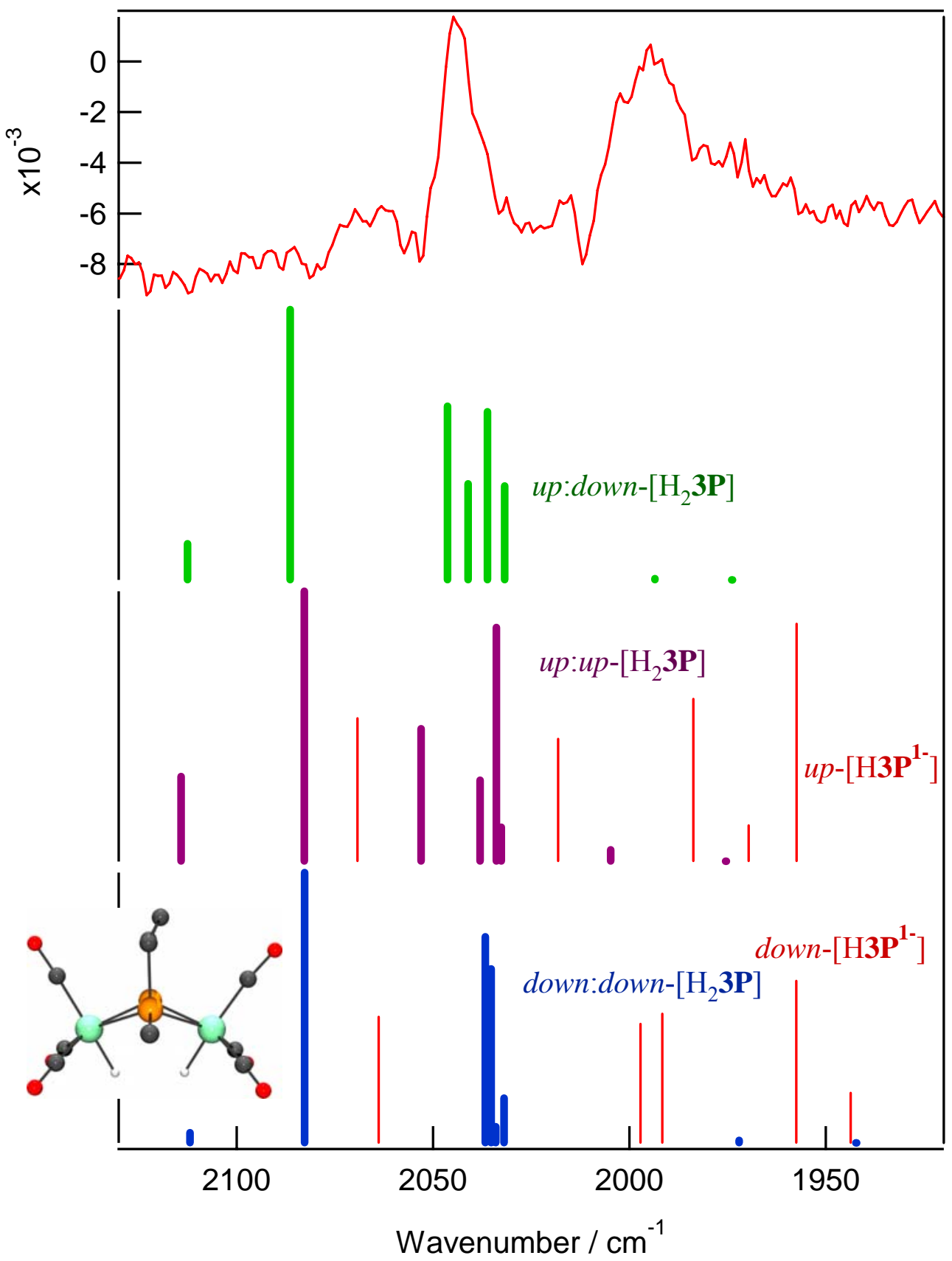

Figure S6. (Top) IR spectra of $\mathrm{H}_{2} 3 P$. Stick figures of calculated frequencies and intensities in the

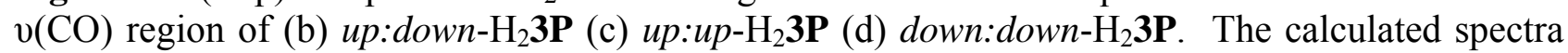
of the singly protonated species are also given (thin lines). 
Table S1 EXAFS models and refinement parameters for $\mathbf{3 P}$ and $\mathbf{3} \mathbf{P}^{\mathbf{2}}$.

Constraints for 3P:

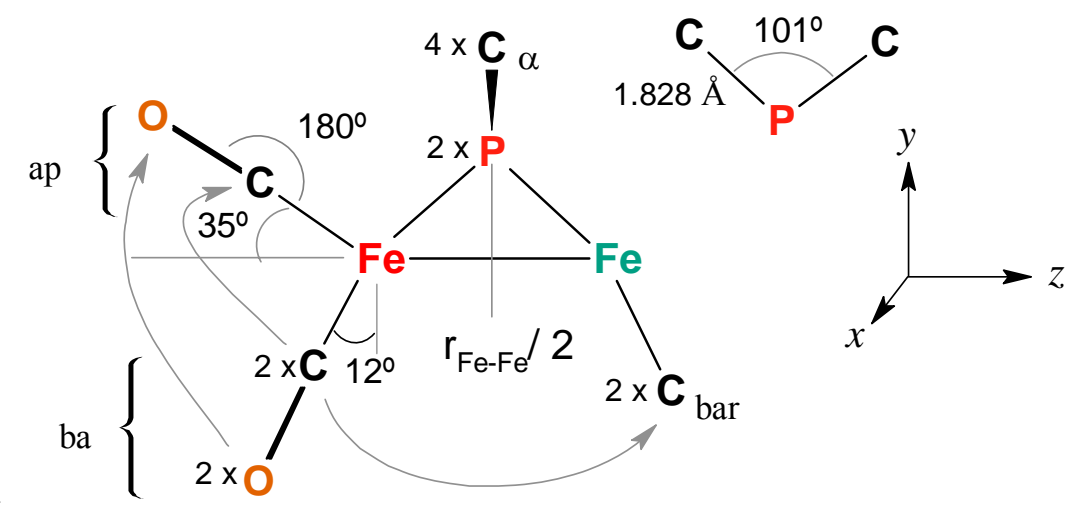

\begin{tabular}{|l|l|l|l|l|l|}
\hline \multicolumn{1}{|c|}{ Atom } & \multicolumn{1}{|c|}{$x$} & \multicolumn{1}{c|}{$y$} & \multicolumn{1}{c|}{$\sigma^{2}$} & \multicolumn{1}{c|}{$\mathrm{n}$} \\
\hline $\mathrm{Fe}$ & 0 & 0 & refined & refined & 1 \\
\hline $\mathrm{P}$ & 0 & refined & $z_{\mathrm{Fe}} / 2$ & refined & 2 \\
\hline $\mathrm{C}_{\alpha}$ & 1.40 & refined & $z_{\mathrm{Fe}} / 2$ & refined & 4 \\
\hline $\mathrm{C}_{\mathrm{ap}}$ & 0 & $-y_{\mathrm{Cba}} \times 0.5864^{a}$ & $y_{\mathrm{Cba}} \times 0.8374^{b}$ & $\sigma_{\mathrm{Cba}}$ & 1 \\
\hline $\mathrm{O}_{\mathrm{ap}}$ & 0 & $-y_{\mathrm{Oba}} \times 0.5864^{a}$ & $y_{\mathrm{Oba}} \times 0.8374$ & $\sigma_{\mathrm{Oba}}$ & 1 \\
\hline $\mathrm{C}_{\mathrm{ba}}$ & 0 & refined & $y_{\mathrm{Cba}} \times \tan \left(12^{\circ}\right)$ & refined & 2 \\
\hline $\mathrm{O}_{\mathrm{ba}}$ & 0 & refined & $y_{\mathrm{Oba}} \times \tan \left(12^{\circ}\right)$ & refined & 2 \\
\hline $\mathrm{C}_{\mathrm{bar}}$ & 0 & $y_{\mathrm{Oba}}$ & $z_{\mathrm{Oba}}$ & $\sigma_{\mathrm{Cba}}$ & 2 \\
\hline
\end{tabular}

${ }^{a} 0.5864=\sin (35) / \cos (12) ;{ }^{b} 0.8374=\cos (35) / \cos (12)$.

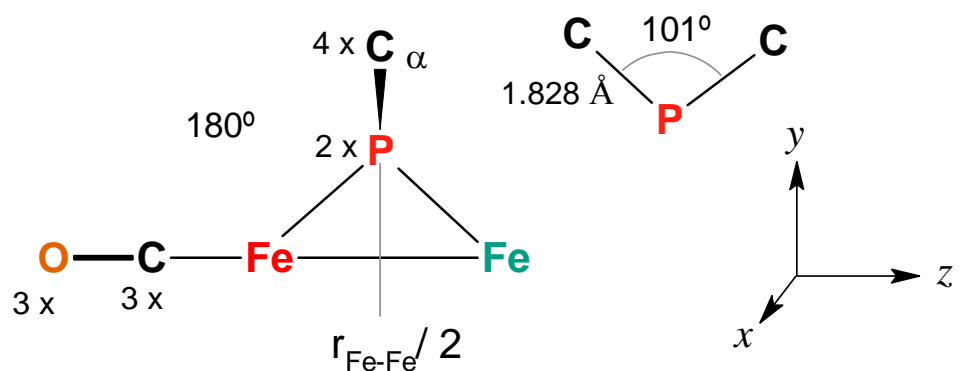

Constraints for $3 \mathbf{P}^{2-}$ :

\begin{tabular}{|l|l|l|l|l|l|}
\hline \multicolumn{1}{|c|}{ Atom } & \multicolumn{1}{c|}{$x$} & \multicolumn{1}{c|}{$y$} & \multicolumn{1}{c|}{$\sigma^{2}$} & \multicolumn{1}{c|}{$\mathrm{n}$} \\
\hline $\mathrm{Fe}$ & 0 & 0 & refined & refined & 1 \\
\hline $\mathrm{P}$ & 0 & refined & $\mathrm{z}_{\mathrm{Fe}} / 2$ & refined & 2 \\
\hline $\mathrm{C}_{\alpha}$ & 1.40 & refined & $z_{\mathrm{Fe}} / 2$ & refined & 4 \\
\hline $\mathrm{C}$ & 0 & 0 & refined & refined & 1 \\
\hline $\mathrm{O}$ & 0 & 0 & refined & refined & 1 \\
\hline
\end{tabular}


Table S2 EXAFS refinement of mixtures.

Fits refining $\mathrm{E}_{0}, \mathrm{~S}_{0}{ }^{2}$ plus an $\mathrm{Fe}-\mathrm{Fe}$ interaction with $\mathrm{DW}$ factor set with a population of $12 \%$. Included in the model are the distances and DW factors that apply to $\mathbf{3 P}$ and $\mathbf{3} \mathbf{P}^{2-}$ where the relative populations are fixed.

\begin{tabular}{|l|l|l||l|l|}
\hline & $40: 12: 48$ ratio & & $44: 12: 44$ ratio & \\
\hline Start: Fe-Fe & Mix 1A & Mix 2 & Mix 1A & Mix 2 \\
\hline 2.8 & $\begin{array}{l}2.779 \\
(5.49,19.11 \%)^{\S}\end{array}$ & $\begin{array}{l}2.785 \\
(4.45,18.07 \%)\end{array}$ & $\begin{array}{l}2.761 \\
(5.18,18.56 \%)\end{array}$ & $\begin{array}{l}2.770 \\
(4.27,17.71 \%)\end{array}$ \\
\hline 2.9 & $\begin{array}{l}2.776 \\
(5.55,19.21 \%)^{\S}\end{array}$ & $\begin{array}{l}2.782 \\
(4.48,18.15 \%)\end{array}$ & $\begin{array}{l}2.758 \\
(5.28,18.73 \%)\end{array}$ & $\begin{array}{l}2.767 \\
(4.33,17.84 \%)\end{array}$ \\
\hline 3.0 & $\begin{array}{l}3.013 \\
(5.94,19.88 \%)\end{array}$ & $\begin{array}{l}3.024 \\
(5.04,19.25 \%)\end{array}$ & $\begin{array}{l}3.037 \\
(5.41,18.97 \%)\end{array}$ & $\begin{array}{l}3.046 \\
(4.78,18.73 \%)\end{array}$ \\
\hline 3.1 & $\begin{array}{l}3.012 \\
(5.98,19.94 \%)\end{array}$ & $\begin{array}{l}3.022 \\
(5.07,19.29 \%)\end{array}$ & $\begin{array}{l}3.035 \\
(5.44,19.02 \%)\end{array}$ & $\begin{array}{l}3.043 \\
(4.79,18.76 \%)\end{array}$ \\
\hline 3.2 & 3.262 & $\begin{array}{l}3.267 \\
(5.21,19.56 \%)\end{array}$ & $\begin{array}{l}3.257 \\
(5.40,18.95 \%)\end{array}$ & $\begin{array}{l}3.261 \\
(4.74,18.67 \%)\end{array}$ \\
\hline 3.3 & $\begin{array}{l}3.301 \\
(6.14,20.21 \%)\end{array}$ & $\begin{array}{l}3.308 \\
(5.25,19.65 \%)\end{array}$ & $\begin{array}{l}3.257 \\
(4.81,18.79 \%)\end{array}$ & $\begin{array}{l}2.261 \\
(4.81,18.79 \%)\end{array}$ \\
\hline 3.4 & $\begin{array}{l}3.262 \\
(6.15,20.21 \%)\end{array}$ & $\begin{array}{l}3.267 \\
(5.25,19.63 \%)\end{array}$ & $\begin{array}{l}3.261 \\
(5.55,19.20 \%)\end{array}$ & $(4.84,18.85 \%)$ \\
\hline
\end{tabular}

${ }^{\S} \chi^{2}, \mathrm{R}_{\mathrm{EXAFS}}$

\begin{tabular}{|l|l||l||l|}
\hline & $42: 12: 46$ ratio & $46: 12: 42$ ratio & $48: 12: 40$ ratio \\
\hline Start: Fe-Fe & Mix 1A & Mix 1A & Mix 1A \\
\hline 2.8 & $2.774(5.39,18.92 \%)$ & $2.766(5.29,18.75 \%)$ & $2.762(5.30,18.77 \%)$ \\
\hline 2.9 & $2.771(5.44,19.01 \%)$ & $2.763(5.33,18.81 \%)$ & $2.759(5.33,18.82 \%)$ \\
\hline 3.0 & $3.013(5.87,19.76 \%)$ & $3.010(5.85,19.72 \%)$ & $3.009(5.91,19.81 \%)$ \\
\hline 3.1 & $3.011(5.91,19.81 \%)$ & $3.032(11.79,19.80 \%)$ & $3.035(5.93,19.86 \%)$ \\
\hline 3.2 & $3.262(5.96,19.90 \%)$ & $3.260(5.88,19.77 \%)$ & $3.259(5.90,19.81 \%)$ \\
\hline 3.3 & $3.303(6.04,20.03 \%)$ & $3.302(5.95,19.88 \%)$ & $3.302(5.97,19.92 \%)$ \\
\hline 3.4 & $3.261(6.04,20.03 \%)$ & $3.260(5.94,19.87 \%)$ & $3.259(5.95,19.89 \%)$ \\
\hline
\end{tabular}


Table S3. Parameters for digital simulation of cyclic voltammogram with 6 equiv. HOTS source program: DigiSim for Windows 95 program version: 3.05 file type: $\mathrm{CV}$ charge transfer reactions:

\begin{tabular}{|l|l|l|l|}
\hline $\begin{array}{l}\text { reaction[1]: } \mathrm{P}+\mathrm{e}= \\
\mathrm{Pm}\end{array}$ & {$[2]: \mathrm{Pm}+\mathrm{e}=\mathrm{P} 2 \mathrm{~m}$} & {$[3]: \mathrm{P} 2 \mathrm{H} 2 \mathrm{~m}+\mathrm{e}=\mathrm{P} 2 \mathrm{H} 3 \mathrm{~m}$} & {$[4]: \mathrm{QHm}+\mathrm{e}=\mathrm{QH} 2 \mathrm{~m}$} \\
\hline
\end{tabular}

homogeneous chemical reactions:

\begin{tabular}{|l|l|l|l|l|}
\hline reaction[1]: & reaction[2]: & reaction[3]: & reaction[4]: & reaction[5]: \\
\hline $\mathrm{P} 2 \mathrm{~m}+\mathrm{H}=\mathrm{PH} 2 \mathrm{~m}$ & $\mathrm{PH} 2 \mathrm{~m}+\mathrm{H}=\mathrm{P} 2 \mathrm{H} 2 \mathrm{~m}$ & $\mathrm{PH} 2 \mathrm{~m}=\mathrm{QH} 2 \mathrm{~m}$ & $\mathrm{P} 2 \mathrm{H} 2 \mathrm{~m}=\mathrm{P}+\mathrm{H} 2$ & $\mathrm{P} 2 \mathrm{H} 3 \mathrm{~m}=\mathrm{Pm}+\mathrm{H} 2$ \\
\hline
\end{tabular}

experimental parameters:

\begin{tabular}{|c|c|c|c|c|c|c|c|c|}
\hline $\mathrm{E}_{\text {start }}$ & $\mathrm{E}_{\text {switch }}$ & $\mathrm{E}_{\text {end }}$ & area & $\mathrm{V}$ & temperature & electrode geometry & diffusion & pre-equilibrium \\
\hline $0.3 \mathrm{~V}$ & $-2.8 \mathrm{~V}$ & $1 \mathrm{~V}$ & $0.008 \mathrm{~cm}^{2}$ & $0.1 \mathrm{~V} / \mathrm{s}$ & $298.2 \mathrm{~K}$ & planar & semi-infinite & enabled for all \\
\hline
\end{tabular}

charge transfer parameters:

\begin{tabular}{|lll|lll|}
\hline E0[1] (V): -1.56 & alpha[1]: 0.5 & $\mathrm{ks}[1](\mathrm{cm} / \mathrm{s}): 0.1$ & $\mathrm{E} 0[2](\mathrm{V}):-1.50$ & alpha[2]: 0.5 & $\mathrm{ks}[2](\mathrm{cm} / \mathrm{s}): 0.1$ \\
\hline E0[3] $(\mathrm{V}):-1.91$ & alpha[3]: 0.5 & $\mathrm{ks}[3](\mathrm{cm} / \mathrm{s}): 0.1$ & $\mathrm{E} 0[4](\mathrm{V}): 0.54$ & alpha[4]: 0.5 & $\mathrm{ks}[4](\mathrm{cm} / \mathrm{s}): 0.1$ \\
\hline
\end{tabular}

chemical reaction parameters:

\begin{tabular}{|c|c|c|c|}
\hline Keq[1]: $1 \mathrm{E}+012$ kf[1]: $1 \mathrm{E}+006$ & $\mathrm{~kb}[1]: 1 \mathrm{E}-006$ & Keq[2]: $1 \mathrm{E}+006$ kf[2]: 5000 & $\mathrm{~kb}[2]: 0.005$ \\
\hline Keq[3]: 10000 & $\mathrm{~kb}[3]: 3 \mathrm{E}-005$ & Keq[4]: $1 \mathrm{E}+005$ kf[4]: 2.4 & $\mathrm{~kb}[4]: 2.4 \mathrm{E}-005$ \\
\hline Keq[5]: $8.225 \mathrm{E}+010$ (TSR) & $\mathrm{E}+010$ & \multicolumn{2}{|c|}{$\mathrm{kb}[5]: 0.12158(\mathrm{TSR}=$ thermodynamically superfluous reaction $)$} \\
\hline
\end{tabular}

species parameters:

\begin{tabular}{|l|l|l|l|l|l|l|l|l|l|l|}
\hline & {$[\mathrm{P}]$} & {$[\mathrm{Pm}]$} & {$[\mathrm{P} 2 \mathrm{~m}]$} & {$[\mathrm{P} 2 \mathrm{H} 2 \mathrm{~m}]$} & {$[\mathrm{P} 2 \mathrm{H} 3 \mathrm{~m}]$} & {$[\mathrm{QHm}]$} & {$[\mathrm{QH} 2 \mathrm{~m}]$} & {$[\mathrm{H}]$} & {$[\mathrm{PH} 2 \mathrm{~m}]$} & {$[\mathrm{H} 2]$} \\
\hline $\mathrm{C}_{\text {anal }}(\mathrm{M} / \mathrm{l})$ & 0.001 & 0 & 0 & 0 & 0 & 0 & 0 & 0.006 & 0 & 0 \\
\hline $\mathrm{C}_{\text {init }}(\mathrm{M} / \mathrm{l})$ & 0.001 & $<10^{-37}$ & $<10^{-72}$ & $<10^{-58}$ & $<10^{-97}$ & $<10^{-59}$ & $<10^{-58}$ & 0.006 & $<10^{-63}$ & $<10^{-51}$ \\
\hline $\mathrm{D}\left(\mathrm{cm}^{2} / \mathrm{s}\right)$ & $1 \mathrm{E}-005$ & $1 \mathrm{E}-005$ & $1 \mathrm{E}-005$ & $1 \mathrm{E}-005$ & $1 \mathrm{E}-005$ & $1 \mathrm{E}-005$ & $1 \mathrm{E}-005$ & $1 \mathrm{E}-$ & $1 \mathrm{E}-005$ & $1 \mathrm{E}-005$ \\
& & & & & & & & 005 & & \\
\hline
\end{tabular}

model parameters:

\begin{tabular}{|c|c|c|c|c|c|c|}
\hline expanding space factor & $\begin{array}{c}\text { noise_leve } \\
1\end{array}$ & r0 minimum & potential step & D/k & iterations & xmax/sqrt(Dt) \\
\hline 0.5 & $0 \mathrm{~A}$ & 20 & $0.005 \mathrm{~V}$ & 50 & 1 & 6 \\
\hline
\end{tabular}


Table S4. Summary of the observed and calculated $v(\mathrm{CO})$ band intensities and wavenumbers.

\begin{tabular}{|c|c|c|c|c|c|}
\hline & $\begin{array}{l}\text { Frequencie } \\
\mathrm{s}\end{array}$ & $\begin{array}{l}\text { Intensitie } \\
\mathrm{s}\end{array}$ & & $\begin{array}{l}\text { Frequencie } \\
\mathrm{s}\end{array}$ & $\begin{array}{l}\text { Intensitie } \\
\mathrm{s}\end{array}$ \\
\hline & 2100.24 & 903.967 & & 2114.09 & 832.91 \\
\hline & 2039.49 & 3895.24 & & 2082.8 & 2648.84 \\
\hline \multirow[t]{7}{*}[3\mathrm{P}_{\mathrm{M}}]{} & 2022.01 & 2319.28 & up:up-[ $\left.\mathrm{H}_{2} \mathbf{3 P}_{\mathrm{M}}\right]$ & 2053.03 & 1298.68 \\
\hline & 2012.87 & 53.401 & & 2037.99 & 795.796 \\
\hline & 2007.96 & 1958.57 & & 2033.87 & 2291.73 \\
\hline & 2000.86 & 418.703 & & 2032.62 & 334.615 \\
\hline & & & & 2004.82 & 114.505 \\
\hline & 2018.65 & 962.673 & & 1975.54 & 2.214 \\
\hline & 1932.66 & 4484.43 & & & \\
\hline \multirow[t]{7}{*}[3\mathbf{P}_{M}{}^{1-}]{} & 1918.1 & 700.017 & & 2111.87 & 123.092 \\
\hline & 1908.66 & 2384.78 & & 2082.67 & 3237.59 \\
\hline & 1905.97 & 3676.1 & & 2036.66 & 2465.37 \\
\hline & 1902.62 & 2974.89 & & 2035.17 & 2083.68 \\
\hline & & & down:down- $\left[\mathrm{H}_{2} 3 \mathrm{P}_{\mathrm{M}}\right]$ & 2034.03 & 193.815 \\
\hline & 1939.63 & 622.625 & & 2031.89 & 534.353 \\
\hline & 1900.77 & 3533.49 & & 1972.11 & 29.9955 \\
\hline \multirow[t]{8}{*}[3\mathrm{P}_{\mathrm{M}}{}^{2-}]{} & 1831.15 & 1863.83 & & 1942.34 & 0.2586 \\
\hline & 1823.47 & 3093.92 & & & \\
\hline & 1805.34 & 2443.17 & & 2117.55 & 119.885 \\
\hline & 1800.14 & 2009.1 & & 2089.51 & 2839.31 \\
\hline & & & & 2045.15 & 2288.83 \\
\hline & 2069.23 & 1403.7 & cis- $\left[\mathrm{H}_{2} \mathrm{DP}\right]$ & 2042.22 & 2323.01 \\
\hline & 2018.14 & 1200.35 & & 2041.71 & 0.3873 \\
\hline & 1983.74 & 1593.21 & & 2037.81 & 220.69 \\
\hline \multirow[t]{7}{*}{$u p-\left[\mathrm{H}_{3} \mathbf{P}_{\mathrm{M}}{ }^{1-}\right]$} & 1969.64 & 351.072 & & 1936.02 & 1.3463 \\
\hline & 1957.45 & 2329.66 & & 1930.81 & 10.1163 \\
\hline & 1871.75 & 1666.81 & & & \\
\hline & 1848.52 & 2495.94 & & 2137.9 & 217.597 \\
\hline & & & & 2108.78 & 2980.32 \\
\hline & 2063.84 & 1508.48 & down:down-[$\left[\mathrm{H}_{2} 3 \mathrm{~S}\right]$ & 2065.73 & 1862.16 \\
\hline & 1997.23 & 1424.6 & & 2063.45 & 555.641 \\
\hline \multirow[t]{8}{*}{ down- $\left[\mathrm{H}_{3} \mathbf{P}_{\mathrm{M}}{ }^{1-}\right]$} & 1991.62 & 1546.82 & & 2060.55 & 1591.59 \\
\hline & 1957.52 & 1941.28 & & 2057.23 & 800.889 \\
\hline & 1943.7 & 597.367 & & 1951.07 & 20.5412 \\
\hline & 1869.52 & 1672.23 & & 1936.61 & 3.2412 \\
\hline & 1842.84 & 2700.17 & & & \\
\hline & 2112.51 & 395.851 & & & \\
\hline & 2086.37 & 2638.18 & & & \\
\hline & 2046.31 & 1713.85 & & & \\
\hline \multirow[t]{5}{*}{ up:down- $\left[\mathrm{H}_{2} \mathbf{3} \mathbf{P}_{\mathrm{M}}\right]$} & 2041.1 & 968.205 & & & \\
\hline & 2036.13 & 1660.76 & & & \\
\hline & 2031.74 & 949.79 & & & \\
\hline & 1993.54 & 60.3257 & & & \\
\hline & 1974 & 49.8443 & & & \\
\hline
\end{tabular}


Table S5: Final Cartesian coordinates for DFT calculated species.

Cartesian coordinates of $\left[\mathbf{3 P}_{\mathbf{M}}\right]$

\begin{tabular}{|c|c|c|c|}
\hline $\mathrm{Fe}$ & 1.29069 & -0.48528 & -0.00025 \\
\hline $\mathrm{Fe}$ & -1.37638 & -0.25833 & 0.00024 \\
\hline$P$ & 0.05113 & 0.78213 & -1.40141 \\
\hline $\mathrm{P}$ & 0.05166 & 0.78206 & 1.40141 \\
\hline $\mathrm{C}$ & 1.54000 & -1.69656 & -1.30599 \\
\hline $\mathrm{C}$ & 2.86554 & 0.33762 & -0.00025 \\
\hline $\mathrm{C}$ & 1.54042 & -1.69659 & 1.30533 \\
\hline $\mathrm{C}$ & -1.87529 & -1.39543 & -1.3029 \\
\hline $\mathrm{C}$ & -2.71447 & 0.90567 & 0.00029 \\
\hline $\mathrm{C}$ & -1.87513 & -1.39532 & 1.30355 \\
\hline $\mathrm{C}$ & 0.17688 & 2.63767 & -1.29576 \\
\hline $\mathrm{C}$ & 0.80986 & 3.16023 & -0.00018 \\
\hline $\mathrm{C}$ & 0.17788 & 2.63755 & 1.29584 \\
\hline $\mathrm{C}$ & -0.01300 & 0.51314 & -3.22500 \\
\hline $\mathrm{C}$ & -0.01204 & 0.51296 & 3.22502 \\
\hline $\mathrm{O}$ & 1.70962 & -2.46869 & -2.13753 \\
\hline $\mathrm{O}$ & 3.89810 & 0.84260 & -0.00012 \\
\hline $\mathrm{O}$ & 1.71034 & -2.46870 & 2.13683 \\
\hline $\mathrm{O}$ & -2.20817 & -2.11396 & -2.1335 \\
\hline $\mathrm{O}$ & -3.57625 & 1.66705 & 0.00019 \\
\hline $\mathrm{O}$ & -2.20799 & -2.11376 & 2.1342 \\
\hline $\mathrm{H}$ & -0.83059 & 3.04525 & -1.43313 \\
\hline $\mathrm{H}$ & 0.77119 & 2.97610 & -2.1503 \\
\hline $\mathrm{H}$ & 0.75583 & 4.25462 & -0.00010 \\
\hline $\mathrm{H}$ & 1.87369 & 2.91131 & -0.00066 \\
\hline $\mathrm{H}$ & 0.77295 & 2.97570 & $2.1499^{7}$ \\
\hline $\mathrm{H}$ & -0.82937 & 3.04539 & 1.4341 \\
\hline $\mathrm{H}$ & -0.14037 & -0.54298 & 3.4601 \\
\hline $\mathrm{H}$ & -0.84910 & 1.06864 & 3.65502 \\
\hline $\mathrm{H}$ & 0.91252 & 0.86432 & 3.68930 \\
\hline $\mathrm{H}$ & -0.85022 & 1.06874 & -3.6547 \\
\hline $\mathrm{H}$ & -0.14123 & -0.54281 & -3.4601 \\
\hline 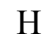 & 0.91142 & 0.86464 & -3.6894 \\
\hline
\end{tabular}

Cartesian coordinates of $\left[3 \mathbf{P}_{\mathbf{M}}{ }^{1-}\right]$

$\begin{array}{cccc}\mathrm{Fe} & -1.55122 & 0.03520 & -0.40501 \\ \mathrm{Fe} & 1.58876 & 0.05331 & -0.29962 \\ \mathrm{P} & 0.00471 & -1.41741 & 0.46650 \\ \mathrm{P} & -0.02064 & 1.28909 & 0.77793 \\ \mathrm{C} & -1.62647 & -0.92164 & -1.90627 \\ \mathrm{C} & -2.96491 & -0.43749 & 0.57202 \\ \mathrm{C} & -2.17451 & 1.52172 & -1.14553 \\ \mathrm{C} & 2.17122 & -1.15856 & -1.46183 \\ \mathrm{C} & 2.85773 & 0.03962 & 0.95419 \\ \mathrm{C} & 1.97073 & 1.43623 & -1.35541 \\ \mathrm{C} & -0.02577 & -1.57236 & 2.33354 \\ \mathrm{C} & -0.59987 & -0.34252 & 3.05495 \\ \mathrm{C} & -0.01720 & 1.01425 & 2.63171 \\ \mathrm{C} & 0.03105 & -3.20142 & -0.03126 \\ \mathrm{C} & 0.05183 & 3.13962 & 0.70324 \\ \mathrm{O} & -1.73283 & -1.55105 & -2.87536 \\ \mathrm{O} & -3.91126 & -0.78516 & 1.14988 \\ \mathrm{O} & -2.58889 & 2.49847 & -1.61322 \\ \mathrm{O} & 2.57134 & -1.95592 & -2.20275 \\ \mathrm{O} & 3.69101 & 0.04845 & 1.76280 \\ \mathrm{O} & 2.25358 & 2.33670 & -2.02996\end{array}$

$\begin{array}{lrrr}\mathrm{H} & 1.00024 & -1.77298 & 2.66346 \\ \mathrm{H} & -0.62354 & -2.45188 & 2.59933 \\ \mathrm{H} & -0.46176 & -0.46886 & 4.13651 \\ \mathrm{H} & -1.67931 & -0.31799 & 2.88733 \\ \mathrm{H} & -0.60607 & 1.81280 & 3.09745 \\ \mathrm{H} & 1.01269 & 1.12759 & 2.99005 \\ \mathrm{H} & 0.09337 & 3.47287 & -0.33415 \\ \mathrm{H} & 0.94229 & 3.50885 & 1.21997 \\ \mathrm{H} & -0.83219 & 3.57986 & 1.17303 \\ \mathrm{H} & 0.90401 & -3.70618 & 0.39233 \\ \mathrm{H} & 0.07789 & -3.28688 & -1.11735 \\ \mathrm{H} & -0.87143 & -3.71004 & 0.31898\end{array}$

Cartesian coordinates of $\left[\mathbf{3 P}_{\mathbf{M}}{ }^{2-}\right]$

$\begin{array}{cccc}\mathrm{Fe} & -1.79372 & 0.07040 & 0.35378 \\ \mathrm{Fe} & 1.84047 & -0.07801 & 0.24997 \\ \mathrm{P} & 0.06682 & 1.29244 & -0.36578 \\ \mathrm{P} & -0.05075 & -1.29559 & -0.38355 \\ \mathrm{C} & -1.59275 & 0.39454 & 2.07442 \\ \mathrm{C} & -2.86286 & 1.18806 & -0.46373 \\ \mathrm{C} & -2.91701 & -1.27547 & 0.32604 \\ \mathrm{C} & 2.91679 & 1.29398 & 0.43838 \\ \mathrm{C} & 2.86643 & -0.95183 & -0.86523 \\ \mathrm{C} & 1.78768 & -0.65273 & 1.91268 \\ \mathrm{C} & 0.03034 & 1.31538 & -2.25146 \\ \mathrm{C} & -0.57965 & 0.03206 & -2.85237 \\ \mathrm{C} & -0.09141 & -1.30840 & -2.26601 \\ \mathrm{C} & 0.10785 & 3.11937 & -0.00502 \\ \mathrm{C} & -0.02382 & -3.12353 & -0.02818 \\ \mathrm{O} & -1.61920 & 0.64883 & 3.21937 \\ \mathrm{O} & -3.57409 & 1.97516 & -0.97700 \\ \mathrm{O} & -3.64077 & -2.19844 & 0.29163 \\ \mathrm{O} & 3.60817 & 2.23420 & 0.54765 \\ \mathrm{O} & 3.57836 & -1.57431 & -1.56919 \\ \mathrm{O} & 1.87672 & -1.08804 & 2.99969 \\ \mathrm{H} & 1.05761 & 1.46409 & -2.60264 \\ \mathrm{H} & -0.56218 & 2.17383 & -2.59549 \\ \mathrm{H} & -0.41730 & 0.03046 & -3.94005 \\ \mathrm{H} & -1.66009 & 0.08197 & -2.69547 \\ \mathrm{H} & -0.77269 & -2.10144 & -2.60212 \\ \mathrm{H} & 0.90783 & -1.56051 & -2.63733 \\ \mathrm{H} & 0.06509 & -3.27400 & 1.04990 \\ \mathrm{H} & 0.83396 & -3.59930 & -0.51405 \\ \mathrm{H} & -0.94314 & -3.60797 & -0.37190 \\ \mathrm{H} & 0.98887 & 3.58871 & -0.45392 \\ \mathrm{H} & 0.15441 & 3.26807 & 1.07605 \\ \mathrm{H} & -0.79205 & 3.61327 & -0.38477\end{array}$

Cartesian coordinates of $u p-\left[\mathrm{H}_{3} \mathbf{P}_{\mathbf{M}}{ }^{1-}\right]$

$\begin{array}{cccc}\mathrm{Fe} & 1.82560 & 0.04946 & 0.13717 \\ \mathrm{Fe} & -1.77600 & -0.02900 & 0.31101 \\ \mathrm{P} & 0.00149 & -1.34323 & -0.37989 \\ \mathrm{P} & -0.07450 & 1.26637 & -0.57500 \\ \mathrm{C} & 1.39696 & 0.27389 & 1.89322 \\ \mathrm{C} & 2.97885 & -1.29674 & 0.31387 \\ \mathrm{C} & 2.92714 & 1.43626 & -0.03277 \\ \mathrm{C} & -1.72838 & -0.65746 & 1.95721\end{array}$




$\begin{array}{lrrr}\mathrm{C} & -2.93114 & -0.79041 & -0.77765 \\ \mathrm{C} & -2.77225 & 1.38009 & 0.66906 \\ \mathrm{C} & -0.07392 & -1.50702 & -2.24502 \\ \mathrm{C} & 0.29861 & -0.22479 & -3.02196 \\ \mathrm{C} & -0.16714 & 1.13003 & -2.44685 \\ \mathrm{C} & 0.09491 & -3.11885 & 0.14981 \\ \mathrm{C} & 0.00134 & 3.10756 & -0.34041 \\ \mathrm{O} & 1.24830 & 0.44660 & 3.01758 \\ \mathrm{O} & 3.70381 & -2.18962 & 0.36681 \\ \mathrm{O} & 3.61700 & 2.33939 & -0.21981 \\ \mathrm{O} & -1.80342 & -1.13008 & 3.02500 \\ \mathrm{O} & -3.72785 & -1.33578 & -1.43995 \\ \mathrm{O} & -3.40118 & 2.33402 & 0.89498 \\ \mathrm{H} & -1.09280 & -1.82528 & -2.48562 \\ \mathrm{H} & 0.59671 & -2.31339 & -2.56397 \\ \mathrm{H} & -0.07689 & -0.31743 & -4.04883 \\ \mathrm{H} & 1.38714 & -0.19248 & -3.10536 \\ \mathrm{H} & 0.43106 & 1.92570 & -2.90717 \\ \mathrm{H} & -1.21349 & 1.32113 & -2.70583 \\ \mathrm{H} & 0.05890 & 3.33392 & 0.72621 \\ \mathrm{H} & -0.90657 & 3.57104 & -0.73649 \\ \mathrm{H} & 0.86777 & 3.54778 & -0.84132 \\ \mathrm{H} & -0.79545 & -3.65477 & -0.19109 \\ \mathrm{H} & 0.12730 & -3.17025 & 1.23972 \\ \mathrm{H} & 0.98247 & -3.61403 & -0.25335 \\ \mathrm{H} & 2.19627 & -0.10258 & -1.32423\end{array}$

Cartesian coordinate of down-[H3P $\left.{ }_{\mathbf{M}}{ }^{1-}\right]$

$\begin{array}{cccc}\mathrm{Fe} & -1.75090 & 0.09423 & -0.34396 \\ \mathrm{Fe} & 1.85896 & -0.00909 & -0.35667 \\ \mathrm{P} & 0.04306 & -1.34036 & 0.18465 \\ \mathrm{P} & 0.13208 & 1.23526 & 0.54079 \\ \mathrm{C} & -2.47177 & 1.58621 & -0.98726 \\ \mathrm{C} & -2.58119 & -1.13194 & -1.33028 \\ \mathrm{C} & -2.81690 & -0.04287 & 1.11159 \\ \mathrm{C} & 1.72806 & -0.31107 & -2.09114 \\ \mathrm{C} & 2.97124 & -1.00053 & 0.57952 \\ \mathrm{C} & 2.95288 & 1.37008 & -0.42690 \\ \mathrm{C} & 0.07936 & -1.63035 & 2.03589 \\ \mathrm{C} & -0.26807 & -0.39518 & 2.89998 \\ \mathrm{C} & 0.17133 & 1.00212 & 2.40391 \\ \mathrm{C} & -0.03734 & -3.07995 & -0.45578 \\ \mathrm{C} & 0.08977 & 3.08821 & 0.41618 \\ \mathrm{O} & -2.88472 & 2.55695 & -1.44721 \\ \mathrm{O} & -3.06224 & -1.92576 & -2.01013 \\ \mathrm{O} & -3.60993 & -0.10442 & 1.94397 \\ \mathrm{O} & 1.72829 & -0.55659 & -3.23447 \\ \mathrm{O} & 3.73013 & -1.68720 & 1.15066 \\ \mathrm{O} & 3.64999 & 2.30319 & -0.46012 \\ \mathrm{H} & 1.08537 & -1.99362 & 2.26629 \\ \mathrm{H} & -0.61550 & -2.43889 & 2.29160 \\ \mathrm{H} & 0.14359 & -0.55116 & 3.90448 \\ \mathrm{H} & -1.34843 & -0.37689 & 3.03872 \\ \mathrm{H} & -0.45951 & 1.75639 & 2.88979 \\ \mathrm{H} & 1.20484 & 1.20904 & 2.69938 \\ \mathrm{H} & 0.08388 & 3.38247 & -0.63487 \\ \mathrm{H} & 0.98624 & 3.50835 & 0.88076 \\ \mathrm{H} & -0.79203 & 3.51026 & 0.90612 \\ \mathrm{H} & 0.84827 & -3.62985 & -0.12532 \\ \mathrm{H} & -0.04211 & -3.06627 & -1.54710 \\ \mathrm{H} & -0.93180 & -3.60162 & -0.10443 \\ & & & \\ & & & \\ & & \end{array}$

$\begin{array}{llll}\mathrm{H} & -0.91591 & 0.23699 & -1.60659\end{array}$

Cartesian coordinates of up:down- $\left[\mathrm{H}_{2} 3 \mathbf{P}_{\mathbf{M}}\right]$

$\begin{array}{crrc}\mathrm{Fe} & -1.86999 & 0.00194 & -0.18161 \\ \mathrm{Fe} & 1.74609 & -0.00770 & -0.37906 \\ \mathrm{P} & -0.02705 & -1.30184 & 0.43854 \\ \mathrm{P} & -0.02096 & 1.32346 & 0.38755 \\ \mathrm{C} & -1.53675 & -0.05017 & -1.97885 \\ \mathrm{C} & -3.03771 & -1.35566 & -0.13140 \\ \mathrm{C} & -3.02922 & 1.36864 & -0.18532 \\ \mathrm{C} & 2.57262 & -1.38471 & -1.17375 \\ \mathrm{C} & 2.74371 & 0.01682 & 1.14097 \\ \mathrm{C} & 2.58030 & 1.33557 & -1.22210 \\ \mathrm{C} & 0.09156 & -1.28308 & 2.30395 \\ \mathrm{C} & -0.28839 & 0.06328 & 2.96818 \\ \mathrm{C} & 0.09577 & 1.38120 & 2.25147 \\ \mathrm{C} & -0.02567 & -3.10800 & 0.04150 \\ \mathrm{C} & -0.01728 & 3.11142 & -0.08502 \\ \mathrm{O} & -1.43027 & -0.11713 & -3.11600 \\ \mathrm{O} & -3.76140 & -2.23831 & -0.03354 \\ \mathrm{O} & -3.75051 & 2.25628 & -0.12196 \\ \mathrm{O} & 3.04251 & -2.27491 & -1.71965 \\ \mathrm{O} & 3.41980 & 0.03145 & 2.06510 \\ \mathrm{O} & 3.05472 & 2.20318 & -1.79954 \\ \mathrm{H} & 1.10795 & -1.58642 & 2.57137 \\ \mathrm{H} & -0.57459 & -2.05911 & 2.69513 \\ \mathrm{H} & 0.13935 & 0.08221 & 3.97654 \\ \mathrm{H} & -1.37050 & 0.06793 & 3.10589 \\ \mathrm{H} & -0.56891 & 2.17402 & 2.61012 \\ \mathrm{H} & 1.11259 & 1.69277 & 2.50739 \\ \mathrm{H} & -0.04931 & 3.20692 & -1.17171 \\ \mathrm{H} & 0.88625 & 3.60550 & 0.28091 \\ \mathrm{H} & -0.88899 & 3.61874 & 0.33543 \\ \mathrm{H} & 0.87537 & -3.58677 & 0.43300 \\ \mathrm{H} & -0.05237 & -3.24914 & -1.04031 \\ \mathrm{H} & -0.90017 & -3.59591 & 0.47875 \\ \mathrm{H} & -2.20169 & 0.02612 & 1.29361 \\ \mathrm{H} & 0.93451 & -0.02774 & -1.66302 \\ & & & \end{array}$

Cartesian coordinates of up:up- $\left[\mathrm{H}_{2} 3 \mathbf{P}_{\mathbf{M}}\right]$

$\begin{array}{cccc}\mathrm{Fe} & 1.81877 & -0.00006 & 0.15992 \\ \mathrm{Fe} & -1.83436 & -0.00001 & 0.11690 \\ \mathrm{P} & -0.00329 & -1.31551 & -0.50941 \\ \mathrm{P} & -0.00325 & 1.31633 & -0.50787 \\ \mathrm{C} & 1.51848 & -0.00105 & 1.96015 \\ \mathrm{C} & 2.97678 & -1.36724 & 0.15072 \\ \mathrm{C} & 2.97724 & 1.36674 & 0.15199 \\ \mathrm{C} & -1.59216 & -0.00138 & 1.92545 \\ \mathrm{C} & -2.99170 & -1.36713 & 0.07122 \\ \mathrm{C} & -2.99139 & 1.36740 & 0.07257 \\ \mathrm{C} & -0.03361 & -1.33250 & -2.37718 \\ \mathrm{C} & 0.37920 & 0.00197 & -3.04584 \\ \mathrm{C} & -0.03379 & 1.33558 & -2.37558 \\ \mathrm{C} & -0.02672 & -3.11323 & -0.07206 \\ \mathrm{C} & -0.02652 & 3.11346 & -0.06813 \\ \mathrm{O} & 1.48654 & -0.00201 & 3.10436 \\ \mathrm{O} & 3.69764 & -2.25431 & 0.07734 \\ \mathrm{O} & 3.69854 & 2.25351 & 0.07943 \\ \mathrm{O} & -1.58734 & -0.00265 & 3.07005 \\ \mathrm{O} & -3.70778 & -2.25580 & -0.02661\end{array}$




$\begin{array}{rrrr}\mathrm{O} & -3.70725 & 2.25635 & -0.02443 \\ \mathrm{H} & -1.04281 & -1.63004 & -2.67310 \\ \mathrm{H} & 0.64678 & -2.11647 & -2.72701 \\ \mathrm{H} & -0.00198 & 0.00257 & -4.07283 \\ \mathrm{H} & 1.46681 & 0.00212 & -3.13633 \\ \mathrm{H} & 0.64639 & 2.12012 & -2.72454 \\ \mathrm{H} & -1.04307 & 1.63325 & -2.67109 \\ \mathrm{H} & -0.05657 & 3.23026 & 1.01667 \\ \mathrm{H} & -0.90682 & 3.60056 & -0.49478 \\ \mathrm{H} & 0.86778 & 3.61352 & -0.44793 \\ \mathrm{H} & -0.90698 & -3.59964 & -0.49957 \\ \mathrm{H} & -0.05697 & -3.23152 & 1.01257 \\ \mathrm{H} & 0.86757 & -3.61287 & -0.45240 \\ \mathrm{H} & 2.18972 & 0.00058 & -1.30383 \\ \mathrm{H} & -2.14418 & 0.00062 & -1.36519\end{array}$

Cartesian coordinates of down:down- $\left[\mathrm{H}_{2} 3 \mathbf{P}_{\mathbf{M}}\right]$

$\begin{array}{cccc}\mathrm{Fe} & 1.79861 & 0.00356 & -0.42231 \\ \mathrm{Fe} & -1.82043 & -0.00120 & -0.40944 \\ \mathrm{P} & -0.01470 & 1.30710 & 0.29845 \\ \mathrm{P} & -0.01135 & -1.30624 & 0.29484 \\ \mathrm{C} & 2.62044 & -1.35228 & -1.25120 \\ \mathrm{C} & 2.62289 & 1.36876 & -1.23392 \\ \mathrm{C} & 2.85562 & -0.00940 & 1.06344 \\ \mathrm{C} & -2.67916 & -1.35941 & -1.19819 \\ \mathrm{C} & -2.68232 & 1.35831 & -1.19237 \\ \mathrm{C} & -2.75528 & -0.00482 & 1.15332 \\ \mathrm{C} & -0.06802 & 1.34002 & 2.16577 \\ \mathrm{C} & 0.28007 & -0.00169 & 2.86405 \\ \mathrm{C} & -0.06364 & -1.34287 & 2.16231 \\ \mathrm{C} & -0.03507 & 3.10294 & -0.14035 \\ \mathrm{C} & -0.02704 & -3.10145 & -0.14685 \\ \mathrm{O} & 3.08644 & -2.22648 & -1.82586 \\ \mathrm{O} & 3.09118 & 2.24976 & -1.79613 \\ \mathrm{O} & 3.62862 & -0.02678 & 1.90880 \\ \mathrm{O} & -3.17307 & -2.23487 & -1.74758 \\ \mathrm{O} & -3.17717 & 2.23687 & -1.73595 \\ \mathrm{O} & -3.39293 & -0.00669 & 2.10470 \\ \mathrm{H} & -1.06349 & 1.68995 & 2.45351 \\ \mathrm{H} & 0.63518 & 2.10337 & 2.51504 \\ \mathrm{H} & -0.19456 & -0.00379 & 3.85053 \\ \mathrm{H} & 1.34901 & -0.00004 & 3.06884 \\ \mathrm{H} & 0.64204 & -2.10478 & 2.50975 \\ \mathrm{H} & -1.05783 & -1.69683 & 2.44948 \\ \mathrm{H} & -0.04032 & -3.21457 & -1.23210 \\ \mathrm{H} & -0.91444 & -3.58859 & 0.26478 \\ \mathrm{H} & 0.86243 & -3.59995 & 0.24598 \\ \mathrm{H} & -0.92423 & 3.58685 & 0.27128 \\ \mathrm{H} & -0.04769 & 3.21775 & -1.22543 \\ \mathrm{H} & 0.85260 & 3.60336 & 0.25408 \\ \mathrm{H} & 1.00533 & 0.00810 & -1.71530 \\ \mathrm{H} & -1.06137 & 0.00166 & -1.72419 \\ & & & \\ & & & \end{array}$

Cartesian coordinates of cis- $\left[\mathrm{H}_{2} \mathbf{D P} \mathbf{P}_{\mathbf{M}}\right]$

$\begin{array}{cccc}\mathrm{Fe} & 1.81662 & 0.00000 & -0.14945 \\ \mathrm{Fe} & -1.81680 & 0.00016 & -0.14711 \\ \mathrm{P} & -0.00018 & -1.45294 & -0.20256 \\ \mathrm{P} & 0.00000 & 1.45312 & -0.20181\end{array}$

$\begin{array}{rrrr}\mathrm{C} & 2.95458 & 1.34400 & -0.51253 \\ \mathrm{C} & 2.95432 & -1.34345 & -0.51479 \\ \mathrm{C} & 1.94748 & -0.00109 & 1.66100 \\ \mathrm{C} & -2.95513 & 1.34403 & -0.50924 \\ \mathrm{C} & -2.95507 & -1.34341 & -0.51052 \\ \mathrm{C} & -1.94537 & -0.00058 & 1.66350 \\ \mathrm{C} & 0.00061 & -2.73075 & 1.14241 \\ \mathrm{C} & 0.00089 & 2.72999 & 1.14406 \\ \mathrm{C} & -0.00120 & -2.54434 & -1.69500 \\ \mathrm{C} & -0.00100 & 2.54551 & -1.69352 \\ \mathrm{O} & 3.64642 & 2.21147 & -0.79246 \\ \mathrm{O} & 3.64569 & -2.21051 & -0.79712 \\ \mathrm{O} & 2.03755 & -0.00208 & 2.80265 \\ \mathrm{O} & -3.64716 & 2.21143 & -0.78891 \\ \mathrm{O} & -3.64700 & -2.21051 & -0.79133 \\ \mathrm{O} & -2.03399 & -0.00113 & 2.80527 \\ \mathrm{H} & -0.88626 & -3.36578 & 1.06740 \\ \mathrm{H} & 0.88674 & -3.36660 & 1.06557 \\ \mathrm{H} & 0.88763 & 3.36513 & 1.06840 \\ \mathrm{H} & -0.88531 & 3.36595 & 1.06890 \\ \mathrm{H} & -0.00172 & 1.93315 & -2.59487 \\ \mathrm{H} & -0.88721 & 3.18513 & -1.70265 \\ \mathrm{H} & 0.88535 & 3.18491 & -1.70390 \\ \mathrm{H} & -0.88786 & -3.18332 & -1.70493 \\ \mathrm{H} & -0.00105 & -1.93140 & -2.59595 \\ \mathrm{H} & 0.88468 & -3.18440 & -1.70536 \\ \mathrm{H} & 1.55094 & 0.00063 & -1.64993 \\ \mathrm{H} & -1.55301 & 0.00061 & -1.64794 \\ \mathrm{H} & 0.00183 & -2.26018 & 2.12660 \\ \mathrm{H} & 0.00096 & 2.25871 & 2.12791\end{array}$

Cartesian coordinates of down:down-[ $\left[\mathrm{H}_{2} 3 \mathrm{~S}\right]$

$\begin{array}{cccc}\mathrm{Fe} & -1.69750 & -0.40523 & 0.00159 \\ \mathrm{Fe} & 1.71987 & -0.40498 & -0.00174 \\ \mathrm{~S} & 0.02044 & 0.31648 & 1.49749 \\ \mathrm{~S} & 0.01732 & 0.32656 & -1.49258 \\ \mathrm{C} & -2.52357 & -1.26919 & -1.33784 \\ \mathrm{C} & -2.52633 & -1.26674 & 1.34158 \\ \mathrm{C} & -2.86055 & 1.03402 & -0.00502 \\ \mathrm{C} & 2.58449 & -1.22710 & -1.34561 \\ \mathrm{C} & 2.58812 & -1.23303 & 1.33630 \\ \mathrm{C} & 2.75654 & 1.12246 & -0.00050 \\ \mathrm{C} & 0.10550 & 2.16254 & 1.34049 \\ \mathrm{C} & -0.32780 & 2.80127 & 0.01240 \\ \mathrm{C} & 0.10126 & 2.17176 & -1.32166 \\ \mathrm{O} & -2.98522 & -1.86033 & -2.19772 \\ \mathrm{O} & -2.98963 & -1.85547 & 2.20214 \\ \mathrm{O} & -3.72656 & 1.77846 & -0.02132 \\ \mathrm{O} & 3.07166 & -1.79271 & -2.20878 \\ \mathrm{O} & 3.07775 & -1.80196 & 2.19586 \\ \mathrm{O} & 3.49432 & 1.99362 & -0.00287 \\ \mathrm{H} & 1.13191 & 2.42710 & 1.59909 \\ \mathrm{H} & -0.52823 & 2.55939 & 2.13692 \\ \mathrm{H} & 0.04166 & 3.83512 & 0.01522 \\ \mathrm{H} & -1.41221 & 2.89691 & 0.01484 \\ \mathrm{H} & -0.53532 & 2.57386 & -2.11317 \\ \mathrm{H} & 1.12655 & 2.43900 & -1.58181 \\ \mathrm{H} & -0.90973 & -1.69807 & -0.00007 \\ \mathrm{H} & 0.97491 & -1.72555 & -0.00509\end{array}$

